\title{
Scrutinizing the Biomarkers for the Neglected Chagas Disease: How Remarkable!
}

\author{
Rosa T. Pinho', Mariana C. Waghabi², Fabiola Cardillo ${ }^{3}$, José Mengel ${ }^{1,4}$ \\ and Paulo R. Z. Antas ${ }^{1 *}$ \\ 'Laboratório de Imunologia Clínica, Instituto Oswaldo Cruz, FIOCRUZ, Rio de Janeiro, Brazil, ${ }^{2}$ Laboratório de Genômica \\ Funcional e Bioinformática, Instituto Oswaldo Cruz, FIOCRUZ, Rio de Janeiro, Brazil, ${ }^{3}$ Centro de Pesquisa Gonçalo Muniz, \\ FIOCRUZ, Salvador, Brazil, "4Faculdade de Medicina de Petropolis (FMP-FASE), Petrópolis, Brazil
}

\section{OPEN ACCESS}

Edited by: Alexandre Morrot, Federal University of Rio de Janeiro, Brazil

Reviewed by:

Marisa Mariel Fernandez, University of Buenos Aires, Argentina Celio Geraldo Freire De Lima, Federal University of Rio de Janeiro, Brazi

*Correspondence:

Paulo R. Z. Antas pzuquim@ioc.fiocruz.br

Specialty section: This article was submitted to Microbial Immunology, a section of the journal

Frontiers in Immunology

Received: 23 June 2016 Accepted: 28 July 2016 Published: 11 August 2016

Citation:

Pinho RT, Waghabi MC, Cardillo F, Mengel J and Antas PRZ (2016)

Scrutinizing the Biomarkers for the Neglected Chagas

Disease: How Remarkable!

Front. Immunol. 7:306. doi: 10.3389/fimmu.2016.00306
Biomarkers or biosignature profiles have become accessible over time in populationbased studies for Chagas disease. Thus, the identification of consistent and reliable indicators of the diagnosis and prognosis of patients with heart failure might facilitate the prioritization of therapeutic management to those with the highest chance of contracting this disease. The purpose of this paper is to review the recent state and the upcoming trends in biomarkers for human Chagas disease. As an emerging concept, we propose a classification of biomarkers based on plasmatic-, phenotype-, antigenic-, genetic-, and management-related candidates. The available data revisited here reveal the lessons learned thus far and the existing challenges that still lie ahead to enable biomarkers to be employed consistently in risk evaluation for this disease. There is a strong need for biomarker validation, particularly for biomarkers that are specific to the clinical forms of Chagas disease. The current failure to achieve the eradication of the transmission of this disease has produced determination to solve this validation issue. Finally, it would be strategic to develop a wide variety of biomarkers and to test them in both preclinical and clinical trials.

Keywords: Chagas disease, biomarkers for immune responsiveness, human experimentation, clinical forms, mini review

\section{INTRODUCTION}

Population-based studies have identified a range of biomarkers that indicate exposure to, effects of, and genetic susceptibility for different pathogen-related diseases. These biomarkers could potentially be applied for diagnostic and prognostic purposes in human Chagas disease. The available data reveal the lessons learned to date and the current challenges that still remain to enable biomarkers to be employed reliably in risk evaluation for this disease. Our main purpose here is to revisit the current evidence and future trends in biomarker research for human Chagas disease. And due to recent, elegant systematic reviews focusing on this important topic (see below), we instead present here a literature review.

Chagas disease, or American trypanosomiasis, is caused by the etiological agent Trypanosoma cruzi and affects at least eight million people in Central and South America (1). The morbidity is high. The acute phase of infection is followed by an asymptomatic phase, but $\sim 30 \%$ of infected patients develop a symptomatic, chronic phase that is characterized by either severe cardiac or digestive forms of Chagas disease $(2,3)$. Hence, the identification of consistent and reliable indicators 
of Chagas disease pathology, namely biomarkers or biosignature profiles, might facilitate the prioritization of management to those with a chance of contracting Chagas disease. Biomarker candidates might be engaged for the determination of some forms of Chagas disease. It is difficult to predict features related to morbidity and mortality as well as disease evolution. This information would assist in the supervision, decision-making, and follow-up related to this complex disease. Hence, there is a need to discover simple and quantifiable biomarkers that are more reliable than conventional screening methods and can be applied to support the diagnosis and prognosis of patients with heart failure (4).

A total of two systematic reviews of prospective biomarker targets during the course of therapeutic chronic Chagas disease underlined the requirement for the development of unique biomarkers to assess prompt responses to therapeutic management of the disease $(5,6)$. There are several studies that are investing in new diagnostic approaches to cure Chagas disease, particularly regarding the identification of disease biomarkers. Data from forthcoming studies will assist the categorization of patients in terms of clinical aspects for initial follow-ups (6).

Some blood-derived biomarkers that have demonstrated the capacity to predict the progression of early Chagas disease cardiomyopathy have been engaged to assess the value of antiparasitic drugs and to identify initial cardiac and gastrointestinal injuries in asymptomatic patients. Nevertheless, future studies with extended follow-ups are required to establish biomarkers that are able to assess clinical or parasitological cures following therapy (6).

Biomarkers might be categorized based on biochemical configurations and major biological activities, such as inflammation and cellular injury biomarkers, metabolic biomarkers, prothrombotic biomarkers, and antigenic biomarkers (i.e., specific antigens of the parasite). Conversely, we propose a different classification. Table 1 and the next sections of this review summarize the recent data related to biomarker research. Once the scientific data become more readily available, the future identification of critical and consistent biomarker candidates for human Chagas disease should be simplified.

\section{PLASMATIC-RELATED CANDIDATES}

Effectively treated and cured chagasic patients may be identified based on their patterns of circulating biomarkers (7). Recently, studies have indicated that serum markers, such as $\mathrm{A}$ - and B-type natriuretic peptides (ANP and BNP, respectively), N-terminal pro-BNP, troponin I, TGF- $\beta$, MMP-2, and TIMP-1 and -2 , are higher during the severe stages of Chagas disease and represent cardiac damage and inflammation. However, several candidates are not disease specific. Hitherto, the levels of these aforementioned natriuretic peptides have been found to be higher in Chagas disease patients with cardiomyopathy than in those with different forms or other etiologies. Moreover, natriuretic peptide levels are increased in asymptomatic chagasic patients who exhibit no signs of ventricular dysfunction. Hence, natriuretic peptides showed a high predictive value for evaluated outcomes $(4,8)$. Both of the pioneering studies were performed in Brazilian cohorts. According to the data from these studies, BNP is comparable to echocardiogram in terms of the assessment of cardiological patients. To reinforce those prior findings, another study performed in an independent setting found that $\mathrm{BNP}$, pro-BNP, creatine kinase (CK)-MB, and MMP-2 have high predictive values for short-term mortality even in the presence of a decreased ejection fraction and other clinical signs of congestive heart failure, which were all found to be associated with severe chagasic cardiomyopathy in a Bolivian cohort (9).

TABLE 1 | Summary of biomarker investigations related to Chagas disease.

\begin{tabular}{|c|c|c|c|c|}
\hline Study & Source & Biomarker name & Result & Reference \\
\hline Experimental, parasitemia-specific & Antigenic & Aptamer & Increased levels & $(39,40)$ \\
\hline Chagasic cardiomyopathy & Genetic & CCL2 and MAL/TIRAP & Increased susceptibility & $(41)$ \\
\hline Chagasic cardiomyopathy & Genetic & CCR5 & Protection & (41) \\
\hline Chagasic cardiomyopathy & Phenotype & CD15s+ Treg cells & Protection & $(35,36)$ \\
\hline Chagasic cardiomyopathy & Phenotype & CD27+CD28+CD8+ T cells & Protection & $(37,38)$ \\
\hline Non-specific & Plasmatic & TIMP-1 and TIMP-2 & Increased levels & $(5,6)$ \\
\hline Non-specific & Plasmatic & Troponin I & Increased levels & $(5,6)$ \\
\hline Non-specific & Plasmatic & TGF- $\beta$ & Increased levels & $(5,6)$ \\
\hline Asymptomatic & Plasmatic & IL-10 & Increased levels & $(11)$ \\
\hline Non-specific & Plasmatic & APOA1 & Decreased levels & $(7)$ \\
\hline Non-specific & Plasmatic & Fibronectin & Increased levels & (7) \\
\hline Asymptomatic & Plasmatic & MMP-2 & Increased levels & (14) \\
\hline Chagasic cardiomyopathy & Plasmatic & MMP-9 & Increased levels & (14) \\
\hline Chagasic cardiomyopathy & Plasmatic & $\begin{array}{l}\text { ANP, BNP, N-terminal pro-BNP, IFN- } \gamma \text {, TNF- } \alpha, \mathrm{IL}-1 \beta \text {, } \\
\text { and IL- } 6\end{array}$ & Increased levels & $(4,8,10,11)$ \\
\hline Chagasic cardiomyopathy & Plasmatic & $\begin{array}{l}\text { miRNA-1, miRNA-133a and }-133 b \text {, and miRNA-208a } \\
\text { and }-208 b\end{array}$ & Decreased levels & (13) \\
\hline Experimental, chagasic cardiomyopathy & Plasmatic & PICP and PIIINP & Increased levels & $(15)$ \\
\hline Experimental, chagasic cardiomyopathy & Plasmatic & Syndecan-4, ICAM-1, and Galectin-3 & Increased levels & (16) \\
\hline Efficacy & Management & KMP11, HSP70, PAR2, and Tgp63 & Increased Ab. levels & $(5,6)$ \\
\hline Efficacy & Management & Antigen 13 and SAPA & Increased Ab. levels & $(5,6)$ \\
\hline Efficacy & Management & Tc24 & Increased Ab. levels & (43) \\
\hline
\end{tabular}


Because the BNP procedure is simple and quick, this biomarker can be used in endemic zones of Chagas disease with limited access to echocardiographic housing facilities. Finally, pro-BNP and T. cruzi DNA as detected by PCR are the only tests that have been found to have independent clinical value for disease staging in concert with electrocardiogram (ECG), echocardiogram, and clinical assessments (10). In a different study, the authors found that cytokine levels are related to cardiac injury in Chagas disease (11). Asymptomatic individuals exhibited high IL-10 levels that were associated with better prognosis. Conversely, IL-1 $\beta$, IL-6, IFN- $\gamma$, and TNF- $\alpha$ reached the highest levels of expression in chagasic patients with cardiomyopathy. Overall, these findings sustain the perception that the balance between regulatory and inflammatory cytokines is associated with different forms of chronic Chagas disease $(11,12)$. Some micro (mi)RNAs, such as miRNA-1, miRNA-133, and miRNA-208, have been demonstrated to be involved with gene regulation properties and specific expression profiles, and imbalances can be found in chagasic cardiomyopathy (13). Recently, Santamaria and colleagues (7) pursued the identification of serum biomarkers that might be used as surrogates of therapeutic management in Chagas disease. APOA1 and specific fragments thereof and one fragment of fibronectin were uncovered. In chagasic samples, these biomarkers, excluding the full-length APOA1, are upregulated. These biomarkers revert to regular levels in $43 \%$ of cured patients. Notably, whenever serum MMP-9 levels are dominant, cardiac remodeling is strengthened and the advance of the cardiac form of Chagas disease is favored. Conversely, when serum MMP-2 levels prevail, patients persist as clinically asymptomatic. These processes might be IL- $1 \beta$ - and TNF- $\alpha$ dependent (14). In a particular model of infection, the cardiac levels of collagen I, III, and IV rise steadily and reach a peak during the chronic phase of Chagas disease. Thereafter, high serum levels of procollagen type I carboxy-terminal propeptide (PICP) and procollagen type III amino-terminal propeptide (PIIINP) are also observed. Given that increased PICP and PIIINP levels may indicate cardiac fibrosis, it is tempting to speculate that both biomarkers are suitable for detecting fibrosis during cardiac remodeling associated with T. cruzi infection (15). ICAM-1, galectin-3, and syndecan-4 have been found to be overexpressed in the hearts of mice chronically infected with T. cruzi (16). High levels of expression of galectin-3 in inflammatory cells have also been uncovered, and these levels are correlated with a decline in inflammation. A reduction in syndecan-4 and ICAM-1 might indirectly reduce cell migration into the myocardium and, thus, decrease inflammation (17). By contrast, in attempts to the uncover critical aspects of TGF- $\beta$ as a candidate with prognostic value, several studies have demonstrated the influence of this anti-inflammatory cytokine on the development of chagasic cardiomyopathy by facilitating parasite cell invasion and its cycle $(18,19)$, improving parasite survival $(20,21)$, inducing exacerbated heart fibrosis and remodeling (22, 23), downregulating cardiac gap junctions (24), and mediating hypertrophy of the surviving cardiomyocytes (25). Increased circulating levels of TGF- $\beta$ are observed in chronic Chagas disease patients $(22,26)$, and its active form is observed in the myocardia of chronic patients $(27,28)$. Moreover, due to the substantial involvement of TGF- $\beta$ in the development of cardiac damage observed in Chagas disease, active compounds targeting TGF- $\beta$ are currently under study as alternative treatments for the symptomatic cardiac form of Chagas disease $(24,29)$. Recently, the major cysteine protease from T. cruzi, cruzipain, has been observed to be capable of directly activating latent TGF- $\beta$, which favors parasite invasion into host cells (30). New therapeutic approaches for Chagas disease using anti-cruzipain compounds would be of beneficial not only due to their trypanocidal effect but also because they indirectly inhibit different TGF- $\beta$ activities that are crucial for the development of Chagas disease. A retrospective study reported evidence supporting the clinical prognostic value of TGF- $\beta$ as a biomarker for Chagas disease by demonstrating an association between its serological levels and clinical outcomes after 10 years of follow-up (31). Accordingly, TGF- $\beta$ has demonstrated prognostic value as an independent predictor of all-cause mortality in patients without heart failure and with an ejection fraction above $45 \%$. The optimal TGF- $\beta$ cutoff for identifying patients who presented with all-cause mortality was $12.9 \mathrm{ng} / \mathrm{ml}$. A further prospective study is clearly necessary to validate these data. Thus, the serological levels of TGF- $\beta$ could be considered one potential biomarker for the outcome of Chagas disease and, moreover, could be used to follow the effects of treatments and interventions.

\section{PHENOTYPE-RELATED CANDIDATES}

First, we would like to propose the regulatory T cell (Treg) axis as a biomarker for Chagas disease progression. As discussed elsewhere, a malfunction of regulatory immune mechanisms may also be involved in the pathogenesis of Chagas disease (32). This malfunction may be due to the action of Treg cells that have the potential to curb effector responses, allow a partially effective anti-parasite immune response, and therefore enable the establishment and maintenance of chronic Chagas infection (32). By contrast, recent findings in humans have demonstrated an increased rate of Treg cells in chagasic patients in the indeterminate chronic phase (free of disease) compared with those with heart damage, which suggests an important role for Treg cells in the control of the inflammatory response during Chagas disease (33). Additionally, using a non-depleting monoclonal antibody to CD25, it was recently demonstrated that Treg cells bearing the CD4+CD25+Foxp3+ phenotype may also help to control the inflammatory immune response in mice that are chronically infected with T. cruzi (34). Therefore, there is a clear indication that the functional activity of Treg cells might be of crucial importance during the chronic phase of the infection due to their potential to decrease tissue damage and pathology. Recently, the expression of CD15s (Sialyl Lewis x) was described to identify the majority of suppressive Treg cells in humans (35). Thus, this biomarker discriminates suppressive from effector CD4+CD25+Foxp3+ T cells in humans (35). Interestingly, a previous study reported that the expression of CD15s is decreased in peripheral blood lymphocytes from patients with severe Chagas disease (36). Although additional studies are urgently required to uncover the critical aspects of both phenotypes, the expression of CD15s in Treg cells may be a reliable biomarker for the prediction of the progression to pathology of chagasic patients. Additionally, 
another distinct phenotype, i.e., fully differentiated memory CD8 T cells (CD27-CD28-) bearing increased CCR7 expression, has been related to Chagas disease outcome (37). This study demonstrated an increase in total effector/memory CD8+ T cells in T. cruzi-infected individuals with mild heart disease compared with otherwise healthy controls. The study was based on the combined expression of CD27 and CD28 as previously proposed by Appay and colleagues (38), being related to a linear differentiation model for memory CD8+ T cells (38). This study suggested different fates of the $\mathrm{T}$ cell lineage, including early, intermediate, and late stages of cell-memory as follows: CD27+CD28+, CD27-CD28+ (or CD27+CD28-), and CD27-CD28- cells, respectively. As has previously been recognized, the proportion of fully differentiated memory (CD27-CD28-) in the total amount of $\mathrm{CD} 8+\mathrm{T}$ cells is increased in mild Chagas disease. Conversely, the frequency of CD27+CD28+CD8+ T cells in the total memory $\mathrm{CD} 8+\mathrm{T}$ cell population decreases as the disease becomes more severe. Albareda and colleagues (37) hypothesized that this pattern could be a consequence of the gradual clonal exhaustion of the CD8+ $\mathrm{T}$ cell population during infection. Analysis of the chemokine receptor CCR7 for lymph node homing (CCR7 expression) also revealed a significant increase in total effector/memory CD8 T cells in subjects with mild heart disease compared with healthy controls (37).

\section{ANTIGENIC-RELATED CANDIDATES}

A correlated ELISA approach to the detection of aptamers in mouse plasma that is highly specific for circulating parasite excreted-secreted antigens (TESA) has been developed for biomarkers of T. cruzi infection $(39,40)$. An aptamer exhibited specific binding to TESA and trypomastigote extract, but it did not bind to self-proteins or Leishmania donovani proteins. Infected mice have exhibited increased levels of aptamer binding compared with control littermates, which suggests this aptamer as a potential candidate for a future biomarker of $T$. cruzi infection. Furthermore, this aptamer might sense circulating biomarkers in both acute and chronic phases of Chagas disease (39). Recently, the same group confirmed that T. cruzi-infected mice have considerably higher biomarker levels than their noninfected counterparts. This study found that the biomarker levels are also diminished upon therapy (40). However, the biomarker levels in the infected and treated group did not decrease entirely and persisted above the assay cutoff point, which suggests that parasitemia was reduced, but a cure was not achieved. The test was capable of distinguishing circulating biomarkers in animals infected with several subpopulations of T. cruzi.

\section{GENETIC-RELATED CANDIDATES}

Genetic markers can provide evidence of the pathogenesis of Chagas disease and also have the potential to be utilized to identify new therapeutic targets. Frade and colleagues (41) studied genetic predispositions that influenced left ventricular ejection fractions in a Brazilian cohort. The authors found that CCL2 and MAL/TIRAP, but not CCR5, were linked to an increased susceptibility to chagasic cardiomyopathy.

\section{MANAGEMENT-RELATED CANDIDATES}

Regardless of most recent advances in drug development, there is virtually no consensus regarding the use of biomarkers to assess the efficacies of new drugs. Between the two main classes of recombinant proteins that are active during distinct ages and stages of Chagas disease, a 16-protein group and a combination of four recombinant proteins, namely KMP11, HSP70, PAR2, and Tgp63, have been identified (5). That combination could potentially serve as biomarkers candidates. Similarly, antibodies against antigen 13, among 5 others comprising the shed acute phase antigen (SAPA), have been demonstrated to be potential markers of cure efficacy [reviewed in Ref. (5)]. Additionally, a complement-mediated lysis test and an ELISA method based on Tc24 have also been created and the latter found to be a reliable candidate for a helpful parasite biomarker $(42,43)$.

\section{FUTURE TRENDS}

Researchers agree that the use of biomarkers in human Chagas disease will foster the progressing steps during the clinical assessment and also assist in the development of consistent diagnostic tools to lessen the time gap between the progression and detection of disease-relevant measures. Additionally, such biomarkers will allow for the prediction of both primary (genetic) and secondary (acquired factors) immunodeficiencies related to individual susceptibility. The deficiency of biomarkers in the prediction of parasitological outcome status and cure represents a main hurdle for the development of new drugs for Chagas disease. Thus, it is crucial to develop a reliable method to assess the cure of this disease. The aforementioned classes of biomarkers could yield uninterrupted longitudinal results related to Chagas disease management. Processes that are commonly used to identify biomarkers cannot be employed as endpoint evaluations in human clinical trials for ethical reasons. Some existing studies aim to develop alternative, emerging applications for biotechnologies based on the results of chagasic biomarker research. Furthermore, data from biomarker discovery research, such as the presence of TESA, could be used in vaccine development. In parasite-challenged vaccinated animals, TESA positivity could be an indication that the immune response was not appropriate to control the infection $(39,40)$. As a result of these struggles, the success of biomarker research in Chagas disease has not yet allowed for a better understanding of the disease risk from the clinical perspective. A range of issues exacerbates this frustration. One such issue is the incomplete validations of many biomarker candidates. Before conducting human trials, it is essential to identify and validate biomarkers that indicate cured patients. It is also necessary to evaluate the risks associated with the use of new agents in larger cohorts; thus, high-throughput biomarker procedures are needed. Ideally, approaching an accurate, rapid, and reliable point-ofcare diagnostic tool in resource-limited, high-burden settings for Chagas disease through evaluation of biomarkers across that clinical spectrum in order to detect relevant pathogen-specific fingerprints will be of communal benefits, which undoubtedly outweigh the costs. The WHO predicted the eradication of 
Chagas disease transmission by the year 2010, but this goal has not yet been achieved. Indeed, the disease is spreading beyond locations in which it was originally endemic (1). It is crucial to understand the partial corroboration of simple biomarker technologies to avoid generating data that exceeds our ability to analyze the applications of novel technologies that are emerging from population studies. Finally, it would be strategic to develop a wide variety of biomarkers and to test them during preclinical and clinical trials.

\section{AUTHOR CONTRIBUTIONS}

$\mathrm{RP}$ and PA conceived and participated in the design and coordination of the manuscript. MW, FC, and JM provided

\section{REFERENCES}

1. WHO. Chagas Disease (American trypanosomiasis) Fact Sheet 340. World Health Organization (2012). Available from: http://www.who.int/mediacentre/ factsheets/fs340/en/index.html

2. Punukollu G, Gowda RM, Khan IA, Navarro VS, Vasavada BC. Clinical aspects of the Chagas' heart disease. Int J Cardiol (2007) 115:279-83. doi:10.1016/j. ijcard.2006.03.004

3. Pinazo MJ, Canas E, Elizalde JI, Garcia M, Gascon J, Gimeno F, et al. Diagnosis, management and treatment of chronic Chagas' gastrointestinal disease in areas where Trypanosoma cruzi infection is not endemic. Gastroenterol Hepatol (2010) 33:191-200. doi:10.1016/j.gastrohep.2009.07.009

4. Wang Y, Moreira Mda C, Heringer-Walther S, Ebermann L, Schultheiss HP, Wessel N, et al. Plasma ACE 2 activity is an independent prognostic marker in Chagas' disease and equally potent as BNP. J Card Fail (2010) 16(2):157-63. doi:10.1016/j.cardfail.2009.09.005

5. Requena-Mendez A, Lopez MC, Angheben A, Izquierdo L, Ribeiro I, Pinazo MJ, et al. Evaluating Chagas' disease progression and cure through bloodderived biomarkers: a systematic review. Expert Rev Anti Infect Ther (2013) 11(9):957-76. doi:10.1586/14787210.2013.824718

6. Pinazo MJ, Thomas MC, Bua J, Perrone A, Schijman AG, Viotti RJ, et al. Biological markers for evaluating therapeutic efficacy in Chagas' disease, a systematic review. Expert Rev Anti Infect Ther (2014) 12(4):479-96. doi:10.15 86/14787210.2014.899150

7. Santamaria C, Chatelain E, Jackson Y, Miao Q, Ward BJ, Chappuis F, et al. Serum biomarkers predictive of cure in Chagas' disease patients after nifurtimox treatment. BMC Infect Dis (2014) 14:302. doi:10.1186/1471-2334-14-302

8. Ribeiro AL, Dos Reis AM, Barros MV, Rocha MOC. Brain natriuretic peptide and left ventricular dysfunction in Chagas' disease. Lancet (2002) 360(9331):461-2. doi:10.1016/S0140-6736(02)09638-1

9. Sherbuk JE, Okamoto EE, Marks MA, Fortuny E, Clark EH, GaldosCardenas G, et al. Biomarkers and mortality in severe Chagas cardiomyopathy. Glob Heart (2015) 10(3):173-80. doi:10.1016/j.gheart.2015.07.003

10. Keating SM, Deng X, Fernandes F, Cunha-Neto E, Ribeiro AL, Adesina B, et al. Inflammatory and cardiac biomarkers are differentially expressed in clinical stages of Chagas disease. Int J Cardiol (2015) 15(199):451-9. doi:10.1016/j. ijcard.2015.07.040

11. Sousa GR, Gomes JA, Fares RC, Damasio MP, Chaves AT, Ferreira KS, et al. Plasma cytokine expression is associated with cardiac morbidity in Chagas' disease. PLoS One (2014) 9(3):e87082. doi:10.1371/journal.pone.0087082

12. Guedes PM, Gutierrez FR, Silva GK, Dellalibera-Joviliano R, Rodrigues GJ, Bendhack LM, et al. Deficient regulatory T cell activity and low frequency of IL-17-producing T cells correlate with the extent of cardiomyopathy in human Chagas' disease. PLoS Negl Trop Dis (2012) 6(4):e1630. doi:10.1371/journal. pntd.0001630

13. Ferreira LR, Frade AF, Santos RH, Teixeira PC, Baron MA, Navarro IC, et al. MicroRNAs miR-1, miR-133a, miR-133b, miR-208a and miR-208b are dysregulated in chronic Chagas disease cardiomyopathy. Int J Cardiol (2014) 175(3):409-17. doi:10.1016/j.ijcard.2014.05.019 helpful discussions and edited the manuscript. All authors wrote, read, and approved the final manuscript.

\section{ACKNOWLEDGMENTS}

The authors are grateful to Mr. Patrick Salinas and to Mrs. Veronica Antas for text editing. We also thank the laboratory staff for their help during the writing of the manuscript.

\section{FUNDING}

This work was partially supported by CNPq-PQ-2 and FAPERJJCNE fellowships (PA), and fully supported by PAPES/CNPq/ FIOCRUZ and Fundação Octacílio Gualberto, Faculdade de Medicina de Petropolis (FMP-FASE).

14. Fares RC, Gomes Jde A, Garzoni LR, Waghabi MC, Saraiva RM, Medeiros NI, et al. Matrix metalloproteinases 2 and 9 are differentially expressed in patients with indeterminate and cardiac clinical forms of Chagas' disease. Infect Immun (2013) 81(10):3600-8. doi:10.1128/IAI.00153-13

15. Castro-Sesquen YE, Gilman RH, Paico H, Yauri V, Angulo N, Ccopa F, et al. Cell death and serum markers of collagen metabolism during cardiac remodeling in Cavia porcellus experimentally infected with Trypanosoma cruzi. PLoS Negl Trop Dis (2013) 7(2):e1996. doi:10.1371/journal.pntd. 0001996

16. Soares MB, de Lima RS, Rocha LL, Vasconcelos JF, Rogatto SR, dos Santos $\mathrm{RR}$, et al. Gene expression changes associated with myocarditis and fibrosis in hearts of mice with chronic chagasic cardiomyopathy. J Infect Dis (2010) 202(3):416-26. doi:10.1086/653481

17. Vasconcelos JF, Souza BS, Lins TF, Garcia LM, Kaneto CM, Sampaio GP, et al. Administration of granulocyte colony-stimulating factor induces immunomodulation, recruitment of $\mathrm{T}$ regulatory cells, reduction of myocarditis and decrease of parasite load in a mouse model of chronic Chagas disease cardiomyopathy. FASEB J (2013) 27(12):4691-702. doi:10.1096/ fj.13-229351

18. Waghabi MC, Keramidas M, Bailly S, Degrave W, Mendonça-Lima L, Soeiro MN, et al. Uptake of host cell transforming growth factor-beta by Trypanosoma cruzi amastigotes in cardiomyocytes: potential role in parasite cycle completion. Am J Pathol (2005) 167(4):993-1003. doi:10.1016/ S0002-9440(10)61189-3

19. Waghabi MC, Keramidas M, Feige JJ, Araujo-Jorge TC, Bailly S. Activation of transforming growth factor beta by Trypanosoma cruzi. Cell Microbiol (2005) 7(4):511-7. doi:10.1111/j.1462-5822.2004.00481.x

20. Silva JS, Twardzik DR, Reed SG. Regulation of Trypanosoma cruzi infections in vitro and in vivo by transforming growth factor beta (TGF-beta). J Exp Med (1991) 174(3):539-45. doi:10.1084/jem.174.3.539

21. dos Reis GA. Evasion of immune responses by Trypanosoma cruzi, the etiological agent of Chagas disease. Braz J Med Biol Res (2011) 44:84-90. doi:10.1590/ S0100-879X2011007500005

22. Araújo-Jorge TC, Waghabi MC, Hasslocher-Moreno AM, Xavier SS, Higuchi ML, Keramidas M, et al. Implication of transforming growth factorbetal in Chagas disease myocardiopathy. J Infect Dis (2002) 186(12):1823-8. doi:10.1086/345882

23. Rossi MA. The pattern of myocardial fibrosis in chronic Chagas' heart disease. Int J Cardiol (1991) 30(3):335-40. doi:10.1016/0167-5273(91)90012-E

24. Waghabi MC, Coutinho-Silva R, Feige JJ, Higuchi Mde L, Becker D, Burnstock G, et al. Gap junction reduction in cardiomyocytes following transforming growth factor-beta treatment and Trypanosoma cruzi infection. Mem Inst Oswaldo Cruz (2009) 104(8):1083-90.

25. Rosenkranz S. TGF-betal and angiotensin networking in cardiac remodeling. Cardiovasc Res (2004) 63(3):423-32. doi:10.1016/j.cardiores.2004.04.030

26. Perez AR, Silva-Barbosa SD, Berbert LR, Revelli S, Beloscar J, Savino W, et al. Immunoneuroendocrine alterations in patients with progressive forms of chronic Chagas disease. J Neuroimmunol (2011) 235(1-2):84-90. doi:10.1016/j.jneuroim.2011.03.010 
27. Araujo-Jorge TC, Waghabi MC, Soeiro MN, Keramidas M, Bailly S, Feige JJ. Pivotal role for TGF-beta in infectious heart disease: the case of Trypanosoma cruzi infection and consequent chagasic myocardiopathy. Cytokine Growth Factor Rev (2008) 19(5-6):405-13. doi:10.1016/j. cytogfr.2008.08.002

28. Araujo-Jorge TC, Waghabi MC, Bailly S, Feige JJ. The TGF- $\beta$ pathway as an emerging target for Chagas disease therapy. Clin Pharmacol Ther (2012) 92(5):613-21. doi:10.1038/clpt.2012.102

29. de Oliveira FL, Araujo-Jorge TC, de Souza EM, de Oliveira GM, Degrave WM, Feige JJ, et al. Oral administration of GW788388, an inhibitor of transforming growth factor beta signaling, prevents heart fibrosis in Chagas disease. PLoS Negl Trop Dis (2012) 6(6):e1696. doi:10.1371/journal.pntd. 0001696

30. Ferrao PM, d'Avila-Levy CM, Araujo-Jorge TC, Degrave WM, Gonçalves AS, Garzoni LR, et al. Cruzipain activates latent TGF- $\beta$ from host cells during T. cruzi invasion. PLoS One (2015) 10(5):e0124832. doi:10.1371/journal. pone. 0124832

31. Saraiva RM, Waghabi MC, Vilela MF, Madeira FS, Sperandio da Silva GM, Xavier SS, et al. Predictive value of transforming growth factor- $\beta 1$ in Chagas disease: towards a biomarker surrogate of clinical outcome. Trans $R$ Soc Trop Med Hyg (2013) 107(8):518-25. doi:10.1093/trstmh/trt050

32. Cardillo F, de Pinho RT, Antas PR, Mengel J. Immunity and immune modulation in Trypanosoma cruzi infection. Pathog Dis (2015) 73(9):ftv082. doi:10.1093/femspd/ftv082

33. de Araujo FF, Vitelli-Avelar DM, Teixeira-Carvalho A, Antas PR, Assis Silva Gomes J, Sathler-Avelar R, et al. Regulatory T cells phenotype in different clinical forms of Chagas disease. PLoS Negl Trop Dis (2011) 5(5):e992. doi:10.1371/journal.pntd.0000992

34. Nihei J, Cardillo F, Dos Santos WL, Pontes-de-Carvalho L, Mengel J. Administration of a nondepleting anti-CD25 monoclonal antibody reduces disease severity in mice infected with Trypanosoma cruzi. Eur J Microbiol Immunol (BP) (2014) 4(2):128-37. doi:10.1556/EuJMI.4.2014.2.6

35. Miyara M, Chader D, Sage E, Sugiyama D, Nishikawa H, Bouvry D, et al. Sialyl Lewis $\mathrm{x}(\mathrm{CD} 15 \mathrm{~s})$ identifies highly differentiated and most suppressive FOXP3high regulatory T cells in humans. Proc Natl Acad Sci U S A. (2015) 112(23):7225-30. doi:10.1073/pnas.1508224112

36. Laucella SA, Riarte A, Prado N, Zapata J, Segura EL. alpha 4 integrins and sialyl Lewis $\mathrm{x}$ modulation in chronic Chagas disease: further evidence of persistent immune activation. Scand JImmunol (2001) 53(5):514-9. doi:10.1046/j.1365-3083.2001.00916.x
37. Albareda MC, Laucella SA, Alvarez MG, Armenti AH, Bertochi G, Tarleton RL, et al. Trypanosoma cruzi modulates the profile of memory CD8 $\mathrm{T}$ cells in chronic Chagas disease patients. Int Immunol (2006) 18(3):465-71. doi:10.1093/intimm/dxh387

38. Appay V, Dunbar PR, Callan M, Klenerman P, Gillespie GM, Papagno L, et al. Memory CD8+ T cells vary in differentiation phenotype in different persistent virus infections. Nat Med (2006) 8:379. doi:10.1038/nm0402-379

39. Nagarkatti R, de Araujo FF, Gupta C, Debrabant A. Aptamer based, non-PCR, non-serological detection of Chagas' disease biomarkers in Trypanosoma cruzi infected mice. PLoS Negl Trop Dis (2014) 8(1):e2650. doi:10.1371/ journal.pntd. 0002650

40. de Araujo FF, Nagarkatti R, Gupta C, Marino AP, Debrabant A. Aptamerbased detection of disease biomarkers in mouse models for Chagas drug discovery. PLoS Negl Trop Dis (2015) 9(1):e3451. doi:10.1371/journal.pntd. 0003451

41. Frade AF, Pissetti CW, Ianni BM, Saba B, Lin-Wang HT, Nogueira LG, et al. Genetic susceptibility to Chagas disease cardiomyopathy: involvement of several genes of the innate immunity and chemokine-dependent migration pathways. BMC Infect Dis (2013) 13:587. doi:10.1186/1471-2334-13-587

42. Galvao LM, Nunes RM, Cançado JR, Brener Z, Krettli AU. Lytic antibody titre as a means of assessing cure after treatment of Chagas' disease: a 10 years follow-up study. Trans R Soc Trop Med Hyg (1993) 87(2):220-3. doi:10.1016/0035-9203(93)90501-G

43. Krautz GM, Galvão LM, Cançado JR, Guevara-Espinoza A, Ouaissi A, Krettli AU. Use of a 24-kilodalton Trypanosoma cruzi recombinant protein to monitor cure of human Chagas' disease. J Clin Microbiol (1995) 33(8):2086-90.

Conflict of Interest Statement: The authors declare that the research was conducted in the absence of any commercial or financial relationships that could be construed as a potential conflict of interest.

The reviewer CL and handling Editor declared their shared affiliation, and the handling Editor states that the process nevertheless met the standards of a fair and objective review.

Copyright $\odot 2016$ Pinho, Waghabi, Cardillo, Mengel and Antas. This is an openaccess article distributed under the terms of the Creative Commons Attribution License (CC BY). The use, distribution or reproduction in other forums is permitted, provided the original author(s) or licensor are credited and that the original publication in this journal is cited, in accordance with accepted academic practice. No use, distribution or reproduction is permitted which does not comply with these terms. 